\title{
Treatment Outcome and Prognostic Factors in Children with Medulloblastoma: A Retrospective Study of 53 Children in a Developing Country, Egypt
}

\author{
Nesreen ALI ${ }^{1}$, Ahmed MOUSTAFA ${ }^{1}$, Reem EMAD ${ }^{2}$, Emad EBEID $^{3}$ \\ ${ }^{1}$ Cairo University, National Cancer Institute, Children Cancer Hospital Egypt, Pediatric Oncology and \\ Hematology \\ ${ }^{2}$ Cairo University, National Cancer Institute, Department of Radiation Oncology \\ ${ }^{3}$ Cairo University, National Cancer Institute, Department of Pediatric Oncology and Hematology, Cairo, \\ EGYPT
}

\begin{abstract}
Medulloblastoma (MB) accounts for $20 \%$ of malignant brain tumours of childhood; however, only scarce data are available about paediatric medulloblastoma patients in developing countries. We aimed to assess the outcome and prognostic factors among Egyptian paediatric MB patients and compare them with high-income countries. This is a retrospective study that includes 53 eligible patients diagnosed with MB during the period from January 2008 to December 2013. Median age at diagnosis was 6 years. The majority of our patients (45) were high-risk patients (84.9\%) (15\% of them infantile $\leq 3$ years), whereas 8 patients (15.1\%) were standard-risk patients. The 5-year overall survival (OS) and disease-free survival (DFS) rates were $54.6 \%$ and $74.8 \%$, respectively. The presence of postoperative residual disease, spinal seeding, $\mathrm{M}$ staging, and completion of chemotherapy protocol made a significant difference in survival rate $(p=0.045$ and $p<0.001 ; p=0.021$ and $p<0.001$, respectively). There was no significant difference between patients presenting at ages younger or older than 3 years old regarding survival rate (5-year OS 50.0\% versus $55.2 \%$, respectively). At the end of this study, 29 patients (54.7\%) were alive, 22 patients (41.5\%) had died and two patients (3.8\%) were lost to follow-up. Two patients relapsed after treatment. Patients with advanced stages and incomplete surgical resection had a poorer outcome. Coordinated, multidisciplinary paediatric neuro-oncology teams with better health care facilities, strong supportive care measures, and proper assessment of long-term morbidity are needed to improve the outcome of childhood MB patients in developing countries.
\end{abstract}

Keywords: Medulloblastoma, Childhood, Treatment, Developing countries

\section{INTRODUCTION}

Paediatric brain tumours affect many children worldwide, contributing to $25 \%$ of all diagnosed paediatric cancers annually. The incidence of pediatric brain tumors in developing countries is lower, usually below 20 per million, than in developed countries. This lower incidence in developing countries may be related to a decreased exposure to potential risk factors or may be an underestimation due to underreporting or the restricted availability of modern diagnostic techniques. ${ }^{1}$ Medulloblastoma (MB) is the most common malignant brain tumour of childhood; it accounts for approximately $20 \%$ of paediatric central nervous system neoplasms in children younger than 14 years old. ${ }^{2,3}$ Each year, approximately 500 new cases are diagnosed worldwide.
ORCIDs: Nesreen ALI: Reem EMAD:
0000-0002-9770-6290 0000-0003-3457-8735
Ahmed MOSTAFA: 0000-0001-9354-4362 Emad EBEID:

0000-0003-0336-227X 
However, only scarce data are available about the incidence and overall survival of paediatric medulloblastoma patients in developing countries. Egypt is one of the Middle East countries, with a population of 95 million; children aged 0-14 years represent 26.8 million (33\%), and 8500 of those children are diagnosed with cancer in Egypt every year. The incidence of brain tumours comprises approximately $25 \%$ of them, and $\mathrm{MB}$ represents $18.8 \%$ of brain tumours. ${ }^{4,5}$ Current standard management includes safe maximal surgical resection followed by craniospinal irradiation (CSI) with chemotherapeutic radiosensitisation and then adjuvant chemotherapy. The overall and event-free survival rates depend on many prognostic factors. In developing countries, the treatment setting and socioeconomic status could affect the outcome. ${ }^{6}$ In this study, we investigated the treatment outcome and prognostic factors of Egyptian paediatric medulloblastoma patients, who presented to the paediatric department of the National Cancer Institute (NCI) at Cairo University in Egypt during the period from 2008 to 2013 and were treated with postoperative, adjuvant, risk-adapted radiotherapy (RT) and chemotherapy. The study investigated the impact of this treatment protocol on survival and compared it with treatment in high-income countries.

\section{PATIENTS and METHODS}

This is a retrospective cohort study, which included 53 patients 18 years old or younger diagnosed with medulloblastoma and treated at the National Cancer Institute (NCI) at Cairo University, Egypt, during the period from January 2008 to December 2013. The extent of surgical resection was categorised into the following four groups: gross total resection (GTR), no visible tumour remaining in the surgical field; near-total resection (NTR), removal of more than $95 \%$ but less than $100 \%$ of tumour; subtotal resection (STR), removal of more than $50 \%$ to less than $95 \%$ of tumour; and partial resection (PR), more than $10 \%$ but less than $50 \%$ excision of tumour, biopsy and open surgical removal or closed (e.g., needle) removal of tissue for the purpose of establishing a pathological diagnosis, with tumour removal less than $10 \%$ of the total tu- mour mass. Evaluation of metastatic disease was based on the Chang classification system. ${ }^{7}$

Patients were stratified into standard risk (SR) and high risk (HR). SR patients were defined thus if the patient was older than 3 years with no evidence of metastatic disease and had totally or near-totally resected disease $(<1.5 \mathrm{~cm}$ residual) on postoperative magnetic resonance imaging (MRI) or computed tomography (CT) scan and a non-anaplastic type. Patients older than 3 years with evidence of metastatic disease or residual tumour $\geq 1.5 \mathrm{~cm}$ or with an anaplastic type were considered HR.

For SR patients, maximal safe surgical resection of the tumour was followed by 2340 cGy of craniospinal radiation and $3240 \mathrm{cGy}$ of radiation to the primary tumour site. In addition to vincristine weekly during radiotherapy for a total of eight doses, maintenance chemotherapy was begun, after a 6-week rest period, in the form of CCNU, vincristine, and cisplatin in eight cycles (COG A9961).

For HR patients, maximal safe surgical resection of the tumour was followed by 3600 cGy of craniospinal radiation and 1800-1980 cGy of radiation directly to the primary tumour site in addition to weekly vincristine during radiotherapy for a total of six doses. Maintenance chemotherapy was begun, after a 6-week rest period, in the form of cisplatin (CDDP), vincristine (VCR), and cyclophosphamide in 28-day cycles for a total of 6 cycles. Some HR patients received isotretinoin concurrently with maintenance chemotherapy and continued for six months after completion of chemotherapy (COG ACNS0332).

Patients younger than 3 years at the time of diagnosis were adapted to the infantile protocol (COG P9934), with neoadjuvant chemotherapy to delay radiotherapy for this group of patients to reduce the harm to the developing nervous system. The infantile group included 8 patients, and they were considered HR; they were grouped together.

Data were collected from patients' files and included age, gender, presenting symptom, radiological findings, cerebral spinal fluid (CSF) cytology, surgical details, radiotherapy treatment details, chemotherapy treatment details, complications of treatment, response to treatment, follow-up status and relapse. 


\section{Statistical Analysis}

Statistical analysis was done using IBM SPSS Statistics version 22 (IBM Corp., Armonk, NY, USA). Numerical data were expressed as a mean and standard deviation or median and range as appropriate. Qualitative data were expressed as frequency and percentage. Chi-squared test or Fisher's exact test was used to examine the relationship between qualitative variables. For non-normal distributed quantitative data, the comparison between two groups was made using the Mann-Whitney U test (non-parametric t-test). Survival analysis was done using the Kaplan-Meier method. The log-rank test was used to compare two survival curves. Multivariate analysis was done using the Cox regression method for the significant factors affecting survival on univariate analysis. The hazard ratio (HR) with its $95 \%$ confidence interval $(\mathrm{CI})$ was used for risk estimation. Overall survival rates calculated from the date of diagnosis to date of death from any cause, living patients or patients lost to follow-up were considered censored on last known alive date. Although disease-free survival was calculated for patients who achieved complete remission from the time of complete response to the time of relapse/progression or death, whichever occurred first, DFS patients neither relapsed/progressed nor died were censored at last assessment before the loss to follow-up. All p-values were two-sided. $\mathrm{p}$ values $<0.05$ were considered significant.

\section{RESULTS}

\section{Patients' Demographics and Clinical Characteristics}

Among the 53 patients with medulloblastoma, 8 patients were younger than 3 years $(15 \%) ; 45$ $(85 \%)$ patients were older than or equal to 3 years old, with a median age of 6 years and a range from 6 months to 18 years. Thirty-one patients were males $(58.5 \%)$, and 22 were females $(41.5 \%)$, with a male-to-female ratio of $1.4: 1$. Vomiting was the most frequent presenting symptom encountered in $90.6 \%$ of the patients, followed by a headache, which was detected in $86.8 \%$ of patients, whereas unsteady gait was observed in $54.7 \%$ and cranial nerve involvement was present in $50.9 \%$. Other presenting symptoms such as convulsions and disturbed consciousness level occurred in fewer percentages. Twenty-three patients had M0 disease (43\%), 20 had M1 disease (38\%), 8 patients had M3 disease (15\%), and two had M4 disease (4\%).

\section{Surgical Treatment and Risk Groups}

The majority (45) of our patients were high-risk patients $(84.9 \%) ; 18 \%$ of them were infantile (younger than 3 years), whereas 8 patients $(15.1 \%)$ were standard risk. Surgical resection was attempted for all patients, with gross total resection in $45.3 \%$ (24 patients), subtotal resection in $15.1 \%$ (8 patients), partial resection and near-total resection in $5(9.4 \%)$ and $4(7.5 \%)$ patients, respectively, and biopsy encountered in $22.6 \%$ (12 patients). Fiftyone patients $(96.3 \%)$ required a ventriculoperitoneal shunt (VPS) insertion before surgery. Surgical complications occurred in 4 patients $(7.5 \%)$, including haematoma in three patients and facial palsy in one patient. Histopathological examination showed classic medulloblastoma in 29 (54.7\%) patients. The desmoplastic and nodular variant appeared in $9(17 \%)$ and $7(13.2 \%)$ patients, respectively, whereas anaplastic/large-cell medulloblastoma was found in $8(15.1 \%)$ patients. CSF cytology was positive for malignant cells in $49.1 \%$ of patients, whereas seeding was detected in $15.1 \%$ of patients (M3 only; no M2 was detected). Bone marrow aspirate and biopsy were performed in 40 patients $(75.5 \%)$, and only two patients had bone marrow biopsy positive for malignant, non-haematopoietic cells (M4), whereas a bone scan was done in 35 patients, and all of them were negative.

\section{Radiation Treatment}

Forty-nine $(92.5 \%)$ patients received adjuvant radiotherapy according to risk stratification. One patient received conformal 50 Gy radiotherapy. The median duration between surgery and radiotherapy was 70 days, ranging from 35 to 273 days. Median overall treatment time (OTT) was 51 days. Interruption of radiotherapy for more than 7 days was encountered in $30(56.6 \%)$ patients. 


\section{Chemotherapy Treatment}

All patients received chemotherapy; 31 patients $(58.5 \%)$ completed their chemotherapy protocol, but 22 patients $(41.5 \%)$ did not complete chemotherapy due to death or sepsis.

\section{Response to Treatment}

Thirty-eight patients $(71.7 \%)$ achieved complete response (CR) and 5 patients $(9.4 \%)$ attained partial response; seven patients $(13.2 \%)$ did not respond (stable disease [SD]) and only three patients (5.7\%) showed progressive disease (PD).

\section{Outcome}

The median follow-up time was 38.6 months, ranging from 13.3 to 81.9 months. Twenty-nine patients $(54.7 \%)$ were alive, 2 patients $(3.8 \%)$ were lost to follow-up, and 22 patients $(41.5 \%)$ died. Six patients died from disease, 12 patients died from infection and sepsis and 4 patients died from both disease and sepsis. Two out of 53 patients relapsed after treatment. One patient had a distant relapse (spine) and was treated with radiotherapy; the other patient had a local relapse and was treated with palliative chemotherapy.

\section{Overall Survival}

Regarding the aggregate group estimates, the surviving proportion at 3 and 5 years was $58.5 \%$ and $54.6 \%$, respectively (Figure 1). Factors that affected the overall survival (OS) included the presence of postoperative residual tumour $(\mathrm{p}=0.045)$, spinal seeding $(\mathrm{p}<0.001)$, M staging $(\mathrm{p}=0.021)$, the presence of a headache $(\mathrm{p}=0.006)$, residual lesion detected by MRI post-radiotherapy $(\mathrm{p}=0.020)$ and completing the chemotherapy protocol $(\mathrm{p}<0.001)$ (Table 1). Age, gender, risk stratification, pathology subtypes, radiotherapy-concomitant vincristine and delay of chemotherapy were not statistically significant when tested by covariate analysis to test their effect on the prognosis and survival of medulloblastoma patients with $\mathrm{p}$ value $(0.991,0.984$, $0.077,0.099,0.753$ and 0.197 ) respectively (Figure 2 and 3). There was no significant difference between patients who received HR protocol with

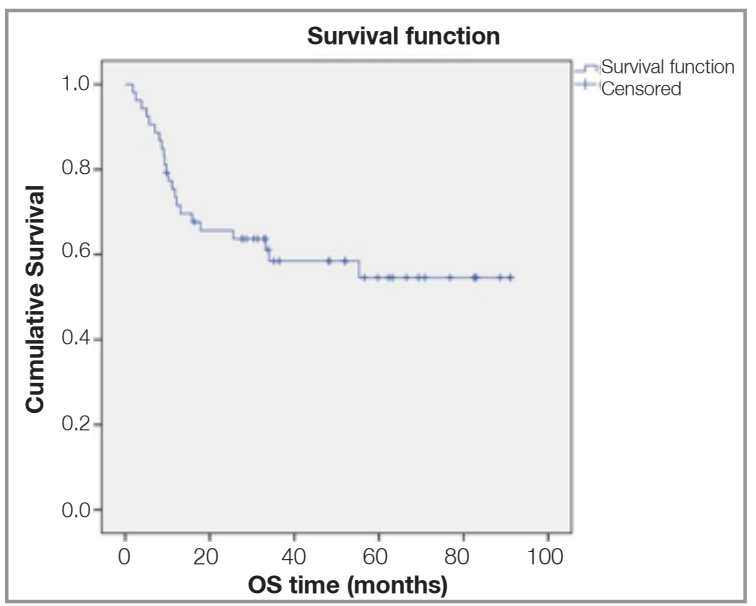

Figure 1. Overall survival

or without isotretinoin as a pro-apoptotic agent in correlation with OS either in the maintenance or continuation cycles with p-value 0.350 and 0.141 , respectively.

\section{Disease-free Survival}

The disease-free survival (DFS) rates at 3 and 5 years were $74.8 \%$. Factors that affected DFS included completing chemotherapy protocol $(\mathrm{p}<$ $0.001)$, headache at presentation $(\mathrm{p}=0.001)$ and visual defect at presentation $(\mathrm{p}=0.025)$ (Table 2$)$.

\section{DISCUSSION}

There is limited data on brain tumours in Egypt, which is one of the biggest developing countries in the Middle East, with a high incidence of paediatric brain tumours. In this retrospective study, we addressed the outcomes, prognostic factors and challenges of treating brain tumours among Egyptian paediatric MB patients who were treated by adjuvant, postoperative, risk-adapted radiotherapy and chemotherapy during the period from January 2008 to December 2013, at the National Cancer Institute of Cairo University, Egypt, and compared it with high-income countries.

Survival of childhood MB has dramatically improved over the past few decades as a result of the adoption of a multimodal treatment approach.8 Recent clinical trials in high-income countries have shown that patients with SR MB who receive 
International Journal of Hematology and Oncology

\begin{tabular}{|c|c|c|c|c|c|c|c|}
\hline & & $\begin{array}{l}\text { No. } \\
\text { deaths }\end{array}$ & $\begin{array}{l}\text { No. of } \\
\text { deaths }\end{array}$ & $\begin{array}{l}\text { \%Surviving at } \\
36 \text { months }\end{array}$ & $\begin{array}{l}\% \text { Surviving } \\
\text { at } 60 \text { months }\end{array}$ & $\begin{array}{l}\text { Median } \\
\text { survival } \\
\text { estimate }\end{array}$ & p \\
\hline Whole group & & 53 & 22 & $58.5 \%$ & $54.6 \%$ & * & \\
\hline \multirow[t]{2}{*}{ Headache } & Present & 46 & 16 & $66.3 \%$ & $61.8 \%$ & * & 0.006 \\
\hline & Absent & 7 & 6 & NR & NR & 11.0 & \\
\hline \multirow{2}{*}{$\begin{array}{l}\text { Postoperative } \\
\text { residual }\end{array}$} & Present & 29 & 16 & $46.6 \%$ & $41.2 \%$ & 34.0 & 0.045 \\
\hline & Absent & 24 & 6 & $73.1 \%$ & $73.1 \%$ & * & \\
\hline \multirow[t]{2}{*}{ Spinal seeding } & Present & 8 & 6 & $N R$ & NR & 8.6 & $<0.001$ \\
\hline & Absent & 44 & 15 & $67.5 \%$ & $63.0 \%$ & * & \\
\hline \multirow[t]{2}{*}{ Stage } & M0 \& M1 & 43 & 16 & $63.8 \%$ & $59.6 \%$ & * & 0.021 \\
\hline & M3 \& M4 & 10 & 6 & $50.0 \%$ & $\mathrm{NR}$ & 9.3 & \\
\hline \multirow{2}{*}{$\begin{array}{l}\text { MRI post } \\
\text { radiotherapy }\end{array}$} & $\mathrm{CR}$ & 27 & 6 & $77.8 \%$ & $77.8 \%$ & * & 0.020 \\
\hline & $\begin{array}{l}\text { Regressive and } \\
\text { stationary }\end{array}$ & 17 & 9 & $44.9 \%$ & $44.9 \%$ & 17.7 & \\
\hline \multirow{2}{*}{$\begin{array}{c}\text { Chemotherapy } \\
\text { completed }\end{array}$} & Yes & 31 & 4 & $89.3 \%$ & $82.9 \%$ & * & $<0.001$ \\
\hline & No & 22 & 18 & $13.3 \%$ & $13.3 \%$ & 9.7 & \\
\hline
\end{tabular}

reduced-dose CSI and adjuvant chemotherapy experience 5-year event-free survival (EFS) greater than $80 \%$, and those with HR MB who receive standard-dose CSI and adjuvant chemotherapy experience 5-year EFS of $66 \%$ to $70.3 \%$. ${ }^{9,10}$ The outcome of MB in low-income countries varies based on the availability of health care resources and the choice of chemotherapy regimens. ${ }^{6}$ In this study, $45(85 \%)$ patients were high-risk, and 8 (15\%) patients were standard-risk. The standard-risk group of patients had a better outcome than those in the high-risk group, with a 5-year OS of $87.5 \%$ and

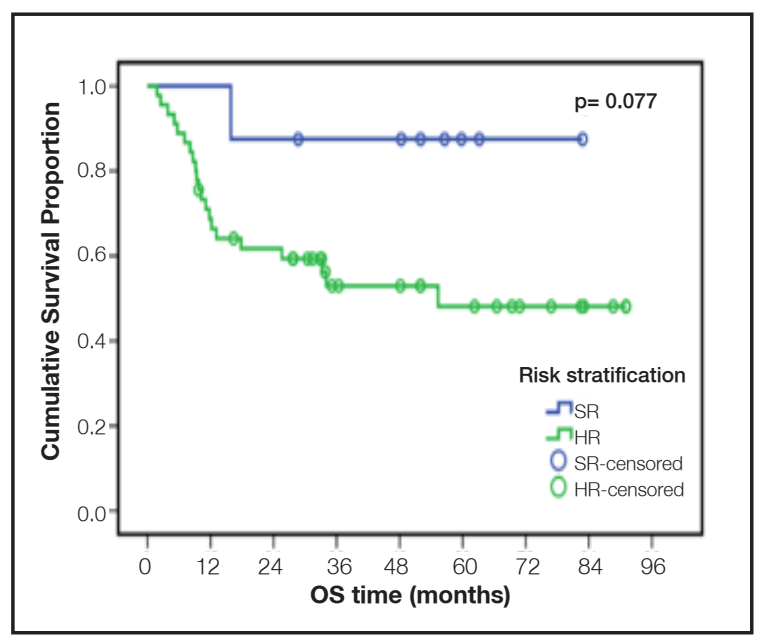

Figure 2. Relation of risk stratification and overall survival
$48.1 \%$, respectively (adjusted $\mathrm{p}=0.077$ ). These rates are comparable to the SR group in highincome countries but considered low for an HR group when compared to those in high-income countries (Table 3). ${ }^{9-12}$ Several reasons may explain this difference. This higher number of HR patients $(85 \%)$ in our study may be explained by the delayed presentation of the patients, relatively advanced stages and improper surgical resection. Our patients underwent their initial surgical intervention elsewhere because of the lack of a paediatric neurosurgical department, and general neurosur-

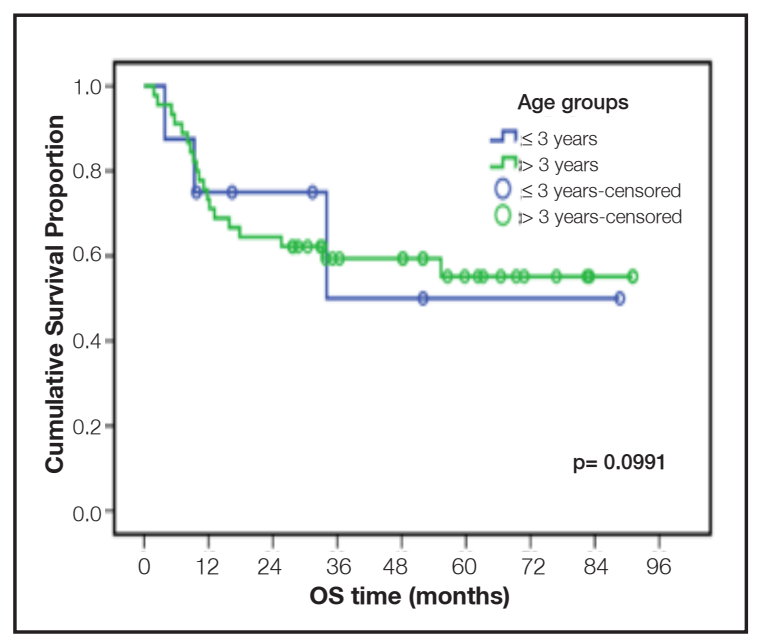

Figure 3. Relation of age and overall survival 


\begin{tabular}{|c|c|c|c|c|c|c|c|}
\hline & & No. & $\begin{array}{l}\text { No. of } \\
\text { events }\end{array}$ & $\begin{array}{l}\text { \%DFS } 36 \\
36 \text { months }\end{array}$ & $\begin{array}{l}\% \text { DFS at } 60 \\
\text { at } 60 \text { months }\end{array}$ & $\begin{array}{l}\text { Median survival } \\
\text { estimate (months) }\end{array}$ & p \\
\hline Whole group & & 37 & 9 & $74.8 \%$ & $74.8 \%$ & * & \\
\hline \multirow[t]{2}{*}{ Headache } & Present & 32 & 5 & $84.3 \%$ & $84.3 .8 \%$ & * & \multirow[t]{2}{*}{0.001} \\
\hline & Absent & 5 & 4 & NR & NR & 21.3 & \\
\hline \multirow{2}{*}{$\begin{array}{c}\text { Chemotherapy } \\
\text { completed }\end{array}$} & Yes & 29 & 2 & $92.9 \%$ & $92.9 \%$ & * & \multirow[t]{2}{*}{$<0.001$} \\
\hline & No & 8 & 7 & $12.5 \%$ & NR & 6.1 & \\
\hline \multirow[t]{2}{*}{ Visual defect } & Present & 17 & 1 & $94.1 \%$ & $94.1 \%$ & * & \multirow[t]{2}{*}{0.025} \\
\hline & Absent & 20 & 8 & $60.0 \%$ & $60.0 \%$ & * & \\
\hline
\end{tabular}

geons performed most of those interventions. As a result, only $45.3 \%$ of paediatric patients had GTR or NTR, and $96.3 \%$ required ventriculoperitoneal shunt (VPS) insertion before surgery.

Our GTR and NTR rate was inferior to that in other developing countries; Menon et al. reported a GTR and NTR rate of $75 \%$ in India, and only $29 \%$ of their patients required a VP shunt. ${ }^{13}$ The limited number of linear accelerators, frequent machine breakdowns, and long waiting lists were significant barriers to commencing radiotherapy (RT) on time (i.e., RT should be given within 4 weeks of surgical resection of MB). Therefore, clinical management was fragmented, and adherence to protocol guidelines was lax. Median overall treatment time (OTT) was 51 days. Interruption of radiotherapy of more than 7 days was encountered in $30(56.6 \%)$ patients. According to the Children's Oncology Group (COG) A9961 phase III trial, it was better to start radiation treatment within 31 days of definitive surgery. ${ }^{14}$ However, this was not achieved for any patient in this study, because the shortest duration between surgical resection and starting RT was 35 days.

In the present study, the 5-year overall survival for those patients who started radiotherapy treatment within 60 days after surgery was $68.8 \%$ compared to $52.8 \%$ for those patients who received their radiotherapy treatment after 60 days from surgery, although it did not show a statistically significant difference (adjusted $\mathrm{p}=0.378$ ), but that may be due to the small sample size. This was in agreement with Taylor et al., who reported that overall survival and EFS were significantly better for patients completing RT within 50 days than for those taking $>50$ days to complete RT (3-year overall survival rate $84.1 \%$ vs. $70.9 \%, p=0.0356,3$-year EFS rate $78.5 \%$ vs. $53.7 \%, \mathrm{p}=0.0092) .{ }^{15}$

The timing of imaging examinations after surgery is important for assessing residual disease and leptomeningeal dissemination. Postoperative imaging of children is often delayed because general anaesthesia may be required to obtain MRIs, and longer imaging time is needed for brain and spine pre- and post-contrast sequences; in addition, there is a waiting list for MRI due to a limited number of machines.

The efficacy of chemotherapy in the treatment of medulloblastoma was assessed previously in two large randomised trials conducted by the International Society of Paediatric Oncology. ${ }^{14,16}$ The addition of chemotherapy for high-risk patients seems to improve their survival and has contributed to a better outcome even in average-risk patients. In this study, all patients were treated with chemotherapy-concurrent chemo-radiotherapy (92.5\%) and adjuvant chemotherapy (100\%)and only eight patients $(15.1 \%)$ were treated with neoadjuvant chemotherapy (before radiotherapy). However, we had 22 patients who did not complete chemotherapy due to sepsis or death. We found that completion of chemotherapy is a crucial factor that affects response to treatment, OS and DFS ( $\mathrm{p}<$ 0.01 ) and confirms the importance of completing the chemotherapy protocol.

Chemotherapy approaches for children younger than 3 years old varied, and the numbers were too 
International Journal of Hematology and Oncology

\begin{tabular}{|c|c|c|c|c|c|c|c|}
\hline & Year & $\begin{array}{l}\text { No. of } \\
\text { patients }\end{array}$ & Treatment & $\begin{array}{l}\text { RTH } \\
\text { CSI }\end{array}$ & $\begin{array}{l}\text { RTH } \\
\text { PF }\end{array}$ & $\begin{array}{l}\text { 5-Year } \\
\text { EFS/PFS } \\
(\%)\end{array}$ & $\begin{array}{l}\text { 5-Year } \\
\text { OS (\%) }\end{array}$ \\
\hline \multicolumn{8}{|l|}{ SR >3 years } \\
\hline Current study & 2018 & 8 & $\begin{array}{l}\text { Standard fractionated RT followed } \\
\text { by } 8 \text { cycles of CDDP, CCNU and VCR }\end{array}$ & 23.4 & 55.8 & & 87.5 \\
\hline Lannering et al. ${ }^{11}$ & 2012 & 340 & $\begin{array}{l}\text { Standard fractionated RT followed by } \\
\text { by } 8 \text { cycles of CDDP, CCNU and VCR }\end{array}$ & 23.4 & 54 & 77 & 87 \\
\hline Packer et al. ${ }^{10}$ & 2006 & 383 & $\begin{array}{l}\text { Weekly VCR during RT followed by } \\
\text { CCNU, CDDP and VCR }\end{array}$ & 23.4 & 55.8 & 82 & 87 \\
\hline \multicolumn{8}{|l|}{ HR >3 years } \\
\hline Current study & 2018 & 45 & $\begin{array}{l}\text { RT followed by } 6 \text { cycles of CDDP, } \\
\text { VCR and cyclophosphamide }\end{array}$ & 36 & 55.8 & 68.5 & 48.1 \\
\hline Tarbell et al. ${ }^{9}$ & 2013 & 224 & $\begin{array}{l}\text { RT, followed by } 3 \text { cycles of CDDP } \\
\text { and Etoposide, and then } 7 \text { cycles of } \\
\text { cyclophosphamide and VCR }\end{array}$ & $35.2-44.0$ & $53.2-54.4$ & 70 & 76.1 \\
\hline Packer et al. ${ }^{12}$ & 1994 & 56 & $\begin{array}{l}\text { Weekly VCR during RT followed by } 8 \\
\text { cycles of VCR, CCNU and CDDP (patien } \\
18 \text { months old included in the analyses) }\end{array}$ & nts. & 55.8 & 85 & \\
\hline
\end{tabular}

small to compare across protocols. The 5-year OS rate was $50 \%$, which was acceptable when compared with those of other low-income countries, but this response might not reflect long-term disease control. This group received definitive surgery followed by chemotherapy, which played an essential role in the treatment of this infantile group because it allowed one to delay the use of radiation for these patients, who received neoadjuvant chemotherapy and reduced the harm to the developing nervous system in this age group..$^{17}$

The most common toxicity in our studied patients was blood toxicity in the form of myelosuppression. Forty-nine patients developed grades 3 and 4 blood toxicity during treatment. The main cause of death in our patients was infection, so good supportive care is critical and considered a crucial factor during treatment, because early detection of any complications will help patients complete their treatment in the prescribed time, especially in developing countries, thus maintaining the overall treatment time (OTT). Moreover, that will confirm the importance of the risk-adapted therapy to adjust the intensity of therapy to the risk of relapse. ${ }^{18}$

With the identification of subgroups based on biological features and their prognostic importance, current treatment challenges include the determination of the optimal dose and timing of radiotherapy, combination of chemotherapy agents, and the development of therapeutic agents that can target specific mutations, thereby contributing to prevention of recurrences while decreasing toxicities and late sequelae for these patients that markedly affect their quality of life. ${ }^{19}$ Unfortunately, lack and unavailability of these tests may be an obstacle inhibiting proper risk stratification and proper treatment.

Cultural barriers and a lack of parental awareness influence treatment adherence in Egypt, and they reflect on delayed presentation of the patients; we had three patients were lost follow-up.

Reasons for treatment abandonment were the intolerance to the adverse effects of chemotherapy, 
sepsis, poor social support during prolonged hospitalisation and lack of housing facilities for families from distant areas; financial issues were not a major factor in treatment because the government subsidises the health care services.

\section{Conclusion}

$\mathrm{MB}$ is one of the most well-studied and frequently encountered central nervous system (CNS) malignancies, with a relatively good response to current treatments. However, the outcome for such patients is poor in developing countries compared to highincome countries. Several steps to overcome the gaps and challenges must be addressed to improve outcomes for children with $\mathrm{MB}$ in low-income countries. Coordinated, multidisciplinary paediatric neuro-oncology teams with better health care facilities, standardised treatment protocols, accurate disease staging and risk stratification and good supportive care during treatment are recommended, because early detection of complications helps patients complete their treatment in the prescribed time, thus maintaining the overall treatment time (OTT) and proper assessment of long-term morbidity. Furthermore, research collaborations with developed countries should be considered to improve the outcome of childhood MB in the future.

\section{REFERENCES}

1. Inskip PD, Tarone RE, Hatch EE, et al. Sociodemographic indicators and risk of brain tumours. Int J Epidemiol 32: 225233, 2003.

2. Ostrom QT, Gittleman H, Liao P, et al. CBTRUS statistical report: primary brain and central nervous system tumours diagnosed in the United States in 2007 - 2011. Neuro Oncol 16(suppl 4), p.iv1-iv63, 2014.

3. Barnholtz-Sloan JS1, Severson RK, Stanton B, et al. Paediatric brain tumours in Non-Hispanics, Hispanics, African Americans, and Asians: differences in survival after diagnosis. Cancer Causes \& Control 16: 587-592, 2005.

4. El Malla H. Improving care of children with cancer in Egypt, Gothenburg University, Clinical Sciences Institute, Clinical Cancer Epidemiology Department of oncology, Graduate Thesis, Gothenburg 2014.

5. El-Gaidi MA. Descriptive epidemiology of paediatric intracranial neoplasms in Egypt. Pediatr Neurosurg 47: 385-395, 2011.
6. Abdelaziz Z, Elsabe B, Fadel S, et al., Outcome and toxicity of Medulloblastoma in Alexandria, EGYPT: 10 years' experience. XVIII International Symposium on Pediatric Neuro-Oncology. Colorado, USA, June 30-July 3, 2018: Pi47.

7. Chang $\mathrm{CH}$, Housepian EM, Herbert C Jr. An operative staging system and a megavoltage radiotherapeutic technic for cerebellar medulloblastomas. Radiology 93: 1351-1359, 1969.

8. Qaddoumi I, Musharbash A, Elayyan M, et al. Closing the survival gap: Implementation of medulloblastoma protocols in a low-income country through a twinning program. Int $\mathrm{J}$ Cancer 122: 1203-1206, 2008.

9. Tarbell NJ, Friedman H, Polkinghorn WR, et al. High-risk medulloblastoma: A Paediatric Oncology Group, randomized trial of chemotherapy before or after radiation therapy (POG 9031). J Clin Oncol 31: 2936-2941, 2013.

10. Packer RJ, Gajjar A, Vezina G, et al. Phase III study of craniospinal radiation therapy followed by adjuvant chemotherapy for newly diagnosed average-risk medulloblastoma. J Clin Oncol 24: 4202-4208, 2006.

11. Lannering B, Rutkowski S, Doz F, et al. Hyperfractionated versus conventional radiotherapy followed by chemotherapy in standard-risk medulloblastoma: Results from the randomized multicenter HIT-SIOP PNET 4 trial. J Clin Oncol 30: 3187-3193, 2012.

12. Packer RJ, Sutton LN, Elterman R, et al. Outcome for children with medulloblastoma treated with radiation and cisplatin, CCNU, and vincristine chemotherapy. J Neurosurg 81: 690-698, 1994.

13. Menon G, Nair S, Muthurethinam T, et al. Medulloblastoma in children: Prognostic factors and predictors of outcome. J Pediatr Neurosci 1: 16-20, 2006.

14. Packer RJ, Zhou T, Holmes E, et al. Survival and secondary tumours in children with medulloblastoma receiving radiotherapy and adjuvant chemotherapy: results of Children's Oncology Group trial A9961. Neuro Oncol 15: 97-103, Jan, 2013.

15. Taylor RE, Bailey CC, Robinson KJ, et al. Impact of radiotherapy parameters on outcome in the International Society of Paediatric Oncology/United Kingdom Children's Cancer Study Group PNET-3 study of preradiotherapy chemotherapy for MO-M1 medulloblastoma. Int J Radiat Oncol Biol Phys 58: 1184-1193, 2004.

16. Gajjar M, Stewart CF, Ellison DW et al. Phase I study of vismodegib in children with recurrent or refractory medulloblastoma: a paediatric brain tumour consortium study. Clin Cancer Res 19: 6305-6312, 2013.

17. Rajagopal R, AbdGhafar S, Ganesan D,et al., Challenges of Treating Childhood Medulloblastoma in a Country with Limited Resources: 20 Years of Experience at a Single Tertiary Center in Malaysia. J Glob Oncol 3: 143-156, 2017. 
18. Packer RJ, Rood BR, MacDonald TJ, et al., Medulloblastoma: present concepts of stratification into risk groups. Paediatr neurosurg 39: 60-67, 2003.

19. Taylor MD, Northcott PA, Korshunov A, et al. Molecular subgroups of medulloblastoma: the current consensus. Acta Neuropathol 123: 465-472, 2012.

\section{Correspondence:}

Nesreen Ali, MD

Cairo University, National Cancer Institute

Department of Pediatric Oncology and Hematology

Pediatric Oncology and Hematology Children Cancer Hospital

Egypt (CCHE57357)

Zahraa Elmaadi, section 7 build 18/39

CAIRO / EGYPT

Tel: $\quad$ 02-01005758221

e-mail: nesreenalinci@hotmail.com

Nesreen.Ali@57357.org 